\title{
Comment
}

Neuroepidemiology 2016;47:210-211

DOI: $10.1159 / 000455182$

\section{Better Care of Stroke Patients, but What about Productivity and Hourly Wages of Stroke Survivors?}

Caroline Arquizan ${ }^{\mathrm{a}}$, Luc Bauchet ${ }^{\mathrm{b}, \mathrm{c}}$

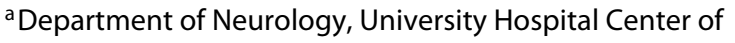
Montpellier, Gui de Chauliac Hospital, CHU Montpellier, and INSERM UMR 894, Paris, b Department of Neurosurgery, University Hospital Center of Montpellier, Gui de Chauliac Hospital, CHU Montpellier and INSERM U 1051, and ' FBTDB ICM, Montpellier, France

In the global scenario, stroke is one of the leading causes of death and disability [1], thereby making acute treatment methods and prevention strategies a global heath priority. Although agestandardised rates of stroke mortality have decreased globally in the past two decades, the absolute number of people who have a stroke every year, stroke survivors, related deaths, and the overall global burden of stroke are great and increasing [2]. The incidence of stroke adjusted to the WHO World standard population ranged from 76 per 100,000 population per year in Australia up to 119 per 100,000 population per year in New Zealand [3]. In the United States, each year, approximately 795,000 people undergo a stroke. About 600,000 of these are first attacks, and 185,000 are recurrent attacks (http://www.strokecenter.org/patients/aboutstroke/stroke-statistics/). Despite the increase in the global burden of stroke, advances are being made, with improved prevention and improved care within the acute phase of stroke (with stroke units, intravenous thrombolysis, endovascular treatment). These treatment methods have shown a significant decrease of death and morbidity after stroke. However, its remains the first cause of disability in adults and the second cause of dementia; poststroke depression affects approximately one third of stroke survivors [4]. Disability is a consequence that is well known after stroke, but other outcomes are under-analyzed (e.g., physical activity and physical fitness levels are low, impacting on health, activity and participation).

In the current issue of Neuroepidemiology, Vyas et al. [5] aim to characterize productivity and factors associated with employability in stroke survivors. The authors used the Canadian Community Health Survey 2011-2012 to identify stroke survivors and employment status. They evaluate the history of stroke and also non-stroke factors associated with employability among stroke survivors between the ages of 18 and 70 years (this upper limit was chosen for comparability of the working population in Canada). They used multivariable logistic models to determine the impact of stroke on employment and factors associated with employability. Heckman models were used to estimate the economic impact of stroke on productivity (number of hours worked/ week and hourly wages). The total number of respondents (18-70 years) and stroke survivors who were included in the study were 91,633 and 923 (1\%) respectively. Stroke survivors were less likely to be employed (adjusted OR $0.39,95 \%$ CI 0.33-0.46) and earned hourly wages, which was $17.5 \%$ (95\% CI 7.7-23.7) lower compared to the general population; however, there was no association between work hours and being a stroke survivor. Among stroke survivors, age greater than 60 , education less than 12th grade, being unmarried and having diabetes and mood disorders were associated with lower odds of employment. This study describes the many known risk factors that validate their sample. One limitation of this well-performed study is the lack of any data about functional data of the stroke survivors.

This study is interesting because it is well known that stroke is a severe condition with high risk of mortality and dependency in about $50 \%$ of survivors, but only few data on the economic burden of stroke is presently available. They used the Heckman model to evaluate the economic impact of stroke and they showed that stroke survivors are less likely than the general population to be employed. Their finding that only $35.3 \%$ of stroke survivors in Canada were employed is consistent with previous studies.

From a clinical point of view, this paper underscores the interests of public health database and the needs to perform populationbased studies. Observation remains one of the major basics of medicine. For medical doctors, the objectives of medical care are that patients could have optimal recovery, good quality of life, and that they could go back to social life; and return to work is a good end point. But, the next step is to know under what conditions patients will be able to return to work. In order to upgrade future works (from clinical point of view), it would be necessary to consider data about recovery after stroke, and level of handicap, for example, with the Rankin score that consists of 6 grades of disability. The low rate of reinsertion is likely attributable to a number of reasons, including not only motor or speech disturbance but also neuropsychological consequences (memory and/or behavioral disorders, etc.), fatigue or mood disorders. When a patient recovers with no disability at all (no symptom), one could expect a better reinsertion, but it is perhaps not always the case, due to other factors, such as the consequence of being a patient with a history of stroke. The information on employment before the stroke is also important to consider.

Afterwards, it would be possible first to answer to the global effectiveness of treatment methods. For example, a large meta-analysis of 5 randomized controlled studies recently showed that an acute endovascular treatment associated with IV thrombolysis in selected patients significantly improved the odds of an excellent outcome, with a better chance to be with no disability at 3 months [6], and this new treatment will be proposed to many stroke patients. Whether these new treatment methods increased performance after stroke, especially among patients who reach good functional recovery, is a matter that has to be evaluated. Second,

\section{KARGER}

E-Mail karger@karger.com

www.karger.com/ned
2017 S. Karger AG, Basel

0251-5350/17/0474-0210\$39.50/0
Luc Bauchet

Department of Neurosurgery

Hôpital Gui de Chauliac - CHU Montpellier, 80 Avenue Augustin Fliche

FR-34295 Montpellier Cedex 5 (France)

E-Mail l-bauchet@chu-montpellier.fr 
questions in the background of the society from which the patients hail are also very important (e.g." "Why do stroke survivors earn a lower hourly wage than people in the general population? Is it due to lower productivity in stroke survivors compared to that of the general population? Or could the long absence due to stroke negatively influence social advancement?"). Further studies are necessary to obtain answers to these questions. Moreover, in case where the "patient" is capable only of less productivity (that probably means that the patient is keeping a disability), does it mean that he deserves to earn less money?

In conclusion, the analysis of outcomes after stroke is essential not only to assess the positive (or negative) impact of a specific medical care but also to raise relevant questions in order to perform further investigations in the ethical and societal spheres.

\section{References}

1 Feigin VL, et al: Atlas of the global burden of stroke (1990-2013): the GBD 2013 study. Neuroepidemiology 2015;45:230-236.

2 Feigin VL, et al: Global and regional burden of stroke during 1990-2010: findings from the Global Burden of Disease Study 2010. Lancet 2014;383: 245-254.

3 Thrift AG, et al: Global stroke statistics. Int J Stroke 2016, in press.

4 Towfighi A, et al: Poststroke depression: a scientific statement for healthcare professionals from the American Heart Association/American Stroke Association. Stroke 2016, in press.

5 Vyas MV, et al: Lost productivity in stroke survivors: an econometrics analysis. Neuroepidemiology 2016;47:164-170.

6 Goyal M, et al: Endovascular thrombectomy after large-vessel ischaemic stroke: a meta-analysis of individual patient data from five randomised trials. Lancet 2016;387:1723-1731. 